\title{
Effects of homocysteine on metabolic pathways in cultured astrocytes
}

\author{
Y. Jin and L. Brennan \\ UCD School of Agriculture, Food Science and Veterinary Medicine, UCD Conway Institute, University College Dublin, \\ Dublin 4, Republic of Ireland
}

Homocysteine is a S-containing non-essential amino acid derived from methionine that is an important risk factor for several neurodegenerative diseases such as Alzheimer's disease and Parkinson's disease ${ }^{(1-3)}$. It plays a key role in the generation of methyl groups required for numerous biochemical reactions. Homocysteine can be either remethylated to methionine by enzymes that require folic acid, and vitamin $B_{12}$ is a cofactor for this remethylation reaction, or catabolised by cystathione $\beta$-synthase, a vitamin $B_{6}$-dependent enzyme, to form cysteine. There is considerable interest in the role of homocysteine in neurodegenerative diseases from a nutrition perspective, as homocysteine levels can be lowered by folic acid and vitamin $\mathrm{B}_{12}{ }^{(4,5)}$.

It has been well established that increased homocysteine levels induce neuronal cell death in a variety of neuronal types ${ }^{(6)}$. However, very few studies have probed the effects of homocysteine in astrocytes. Astrocytes closely interact with neurons to provide structural, metabolic and trophic support and actively participate in modelling neuronal excitability and neurontransmission by controlling the extracellular levels of ions and neurontransmitters ${ }^{(7)}$ As a result, any modulation to astrocyte function could have potential detrimental effects on neuronal-astrocyte cross-talk ${ }^{(8)}$.

The present study investigated for the first time the effects of homocysteine on primary cultures of astrocytes. Primary cultures of rat astrocytes were exposed to $400 \mu \mathrm{M}$-homocysteine for $20 \mathrm{~h}$. Metabolic extracts of cells were prepared following a $4 \mathrm{~h}$ incubation in minimum medium with $5.5 \mathrm{~mm}-\left[\mathrm{U}_{-}{ }^{13} \mathrm{C}\right]$ glucose in the presence or absence of homocysteine. The extracts were analysed using ${ }^{13} \mathrm{C}$ NMR. The expression level of pyruvate dehydrogenase kinase isoform $2(\mathrm{PDK}-2), \mathrm{NAD}(\mathrm{P}) \mathrm{H}$ levels and mitochondrial membrane potential $(\Delta \Psi \mathrm{m})$ responses were investigated following culture in the presence of homocysteine. Metabolomic analysis was performed using ${ }^{1} \mathrm{H}$ NMR spectroscopy and pattern recognition analysis.

\begin{tabular}{|c|c|c|c|c|c|c|c|}
\hline \multirow[b]{2}{*}{ Condition } & \multirow{2}{*}{$\begin{array}{l}\text { PC:PDH ( } \% ; \text { relative } \\
\text { to controls) }\end{array}$} & \multicolumn{2}{|c|}{$\begin{array}{l}\text { PDK-2 protein expression } \\
\text { level }(n \text { 5) }\end{array}$} & \multicolumn{2}{|c|}{$\begin{array}{c}\text { NAD(P)H (fluorescence } \\
\left.(\mathrm{au}) \times 10^{4} ; n 4\right)\end{array}$} & \multicolumn{2}{|c|}{$\Delta \Psi \mathrm{m}\left(\times 10^{7} ; n 4\right)$} \\
\hline & & Mean & SD & Mean & SD & Mean & $\mathrm{SD}$ \\
\hline$\overline{\text { Control }}$ & 100 & 0.43 & 0.05 & 9254 & 1201 & 2.70 & 0.22 \\
\hline Homocysteine & 62 & 0.25 & 0.02 & 15940 & 4462 & 2.13 & 0.29 \\
\hline
\end{tabular}

PC (pyruvate carboxylase): PDH (pyruvate dehydrogenase), flux through pyruvate carboxylase:flux through pyruvate dehydrogenase;

$\mathrm{au}$, arbitrary units.

Following incubation with homocysteine there was a significant decrease (48\%) in PC:PDH, which was the result of an increased flux through PDH. In addition, homocysteine culture resulted in a significant reduction in PDK-2 protein expression $(P=0.01)$. Following stimulation with glucose there was a significant increase in $\mathrm{NAD}(\mathrm{P}) \mathrm{H}$ levels and impaired hyperpolarisation of the mitochondrial membrane in homocysteine-treated cells. Partial least squares discriminate analysis of the metabolic profiles showed that the most discriminating metabolites following homocysteine treatment were choline and hypotaurine. In summary, the results demonstrated that sub-lethal concentrations of homocysteine caused significant metabolic changes and altered mitochondrial function in primary cultures of astrocytes. Future studies will investigate further the detrimental effects of homocysteine on astrocytes and astrocyte-neuronal cross-talk.

1. Bell IR, Edman JS, Selhub J et al. (1992) Acta Psychiatr Scand 86, 386-390.

2. Allen RH, Stabler SP \& Lindenbaum J (1998) Eur J Pediatr 157, Suppl. 2, S122-S126.

3. Miller JW (2000) Nutrition 16, 675-677.

4. Clarke R, Smith AD, Jobst KA et al. (1998) Arch Neurol 55, 1449-1455.

5. Ravaglia G, Forti P, Maioli F et al. (2005) Am J Clin Nutr 82, 636-643.

6. Langmeier M, Folbergrova J, Haugvicova R et al. (2003) Epilepsia 44, 299-304.

7. Escartin C, Valette J, Lebon V et al. (2006) J Neurochem 99, 393-401.

8. Seifert G, Schilling K \& Steinhauser C (2006) Nat Rev Neurosci 7, 194-206. 